\title{
REPERCUSSÕES DA EDUCAÇÃO \\ PERMANENTE NAS PRÁTICAS ASSISTENCIAIS \\ DOS PROFISSIONAIS DE ENFERMAGEM
}

\author{
REPERCUSSIONS OF PERMANENT \\ EDUCATION IN THE ASSISTANCE \\ PRACTICES OF NURSING PROFESSIONALS
}

\section{REPERCUSIONES DE LA EDUCACIÓN CONTINUA EN LAS PRÁCTICAS ASISTENCIALES DE LOS PROFESIONALES DE ENFERMERÍA}

\author{
Maristela Conceição de Jesus ${ }^{1}$ \\ Valdenir Almeida da Silva ${ }^{2}$ \\ Rosana Santos Mota ${ }^{3}$ \\ Juliana Cana Brazil Costa ${ }^{4}$ \\ Andreia Santos Mendes ${ }^{5}$ \\ Marcelle de Jesus Oliveira ${ }^{6}$
}

Como citar este artigo: Jesus MC, Silva VA, Mota RS, Costa JCB, Mendes AS, Oliveira MJ. Repercussões da educação permanente nas práticas assistenciais dos profissionais de enfermagem. Rev baiana enferm. 2019;33:e27555.

Objetivo: analisar as repercussões das atividades de educação permanente nas práticas assistenciais dos profissionais de enfermagem. Método: pesquisa qualitativa, realizada em um hospital universitário de grande porte localizado no município de Salvador, Bahia, Brasil. Foram entrevistados 17 profissionais de enfermagem, entre os meses de setembro e dezembro de 2017. Os dados coletados por meio de entrevista semiestruturada foram processados no software IRAMUTEQ. Resultados: obteve-se um percentual de retenção do texto de 80,3\%, 320 Unidades de Contexto Elementares e 5 classes lexicais. Os entrevistados atribuíram à participação nas atividades de educação permanente um papel relevante, com repercussões positivas para a assistência de enfermagem. Conclusão: a participação em atividades de educação permanente levou à sensibilização sobre as necessidades educativas e, assim, pôde favorecer maior satisfação com o trabalho e melhor qualidade na assistência, visto que os profissionais de enfermagem passaram a sentir-se mais qualificados e atualizados.

Descritores: Educação Permanente. Capacitação em Serviço. Equipe de Enfermagem. Assistência de Enfermagem. Serviços de Saúde.

\footnotetext{
Estudante de Enfermagem. Universidade Federal da Bahia. Salvador, Bahia, Brasil.

Enfermeiro. Doutor em Enfermagem. Enfermeiro da Comissão de Educação Permanente em Enfermagem do Hospital Universitário Professor Edgar Santos. Universidade Federal da Bahia. Salvador, Bahia, Brasil. valdenirenf@yahoo.com

3 Enfermeira. Doutora em Enfermagem. Enfermeira da Comissão de Educação Permanente em Enfermagem do Hospital Universitário Professor Edgar Santos. Universidade Federal da Bahia. Salvador, Bahia, Brasil. rosanal7santos@yahoo.com.br

4 Enfermeira. Chefe da Divisão de Enfermagem do Hospital Universitário Professor Edgar Santos. Universidade Federal da Bahia. Salvador, Bahia, Brasil.

5 Enfermeira. Mestre em Enfermagem. Enfermeira da Comissão de Educação Permanente em Enfermagem do Hospital Universitário Professor Edgar Santos. Universidade Federal da Bahia. Salvador, Bahia, Brasil.

6 Enfermeira. Enfermeira da Comissão de Educação Permanente em Enfermagem do Hospital Universitário Professor Edgar Santos. Universidade Federal da Bahia. Salvador, Bahia, Brasil.
} 
Objective: to analyze the repercussions of the activities of permanent education on nursing assistance practices. Method: qualitative research performed in a large university hospital located in the city of Salvador, Babia, Brazil. 17 nursing professionals were interviewed between September and December 2017. The data collected through a semistructured interview were processed in IRAMUTEQ software. Results: the percentage obtained was $80.3 \%$ text retention, 320 Elementary Context Units and 5 lexical classes. The interviewees attributed to the participation in the activities of permanent education a relevant role, with positive repercussions for nursing care. Conclusion: participation in activities of permanent education led to the awareness of educational needs and, thus, could favor greater satisfaction with work and better quality of care, as the nursing professionals began to feel more qualified and updated.

Descriptors: Permanent Education. In-service Training. Nursing Team. Nursing Care. Health Services.

Objetivo: analizarlas repercusiones de las actividades de educación continua en prácticas asistenciales deprofesionales de enfermería. Método: investigación cualitativa, en hospital universitario de gran porte en Salvador, Babia, Brasil. Se entrevistaron a 17 profesionales de enfermería, entre septiembre y diciembre de 2017. Datos recogidos por medio de entrevista semiestructurada, procesados en el software IRAMUTEQ. Resultados: se obtuvo porcentaje de retención del texto de 80,3\%, 320 Unidades de Contexto Elementales y cinco clases lexicales. Entrevistados atribuyeron papel relevante a la participación en las actividades de educación continua, con repercusiones positivas para atención de enfermería. Conclusión: la participación en actividades de educación continua llevó a la sensibilización sobre necesidades educativas y, así, se pudo favorecer mayor satisfacción con el trabajo y mejor calidad en la atención, ya que los profesionales de enfermería pasaron a sentirse más cualificados y actualizados.

Descriptores: Educación Continua. Capacitación en Servicio. Grupo de Enfermería. Atención de Enfermería. Servicios de Salud.

\section{Introdução}

A educação permanente em saúde tem sido descrita como uma estratégia para ampliar a continuidade da formação dos profissionais de saúde, garantindo acesso a conhecimentos, desenvolvimento de habilidades para a prática, atualização e fortalecimento de competências relevantes para o trabalho ${ }^{(1)}$.

No âmbito do Sistema Único de Saúde (SUS), a necessidade da Educação Permanente em Saúde (EPS) foi fortalecida pela publicação da Portaria n. 1.996, de 20 de agosto de 2007, que lançou as diretrizes para implementação da Política de Educação Permanente. De acordo com tal documento, a EPS constitui-se em uma ferramenta que pode viabilizar a interação aprendizagem-trabalho, por ser desenvolvida no cotidiano dos profissionais e dos serviços. A EPS é implementada com base nas dificuldades e nos problemas enfrentados na realidade, considerando saberes anteriores e promovendo ações cujo objetivo é capacitar profissionais para as práticas aplicadas no trabalho ${ }^{(2)}$.

Uma vez que a qualificação dos profissionais de saúde ${ }^{(3)}$ contribui para o desenvolvimento de novas competências e, portanto, para a excelência nos serviços de saúde ${ }^{(4)}$, a EPS consolida-se como um instrumento para a transformação do profissional em um profundo conhecedor da realidade local. Assim, possibilita a interligação entre o trabalho, a saúde e a educação ${ }^{(5)}$. Autores consideram que a EPS está interligada com a qualidade da assistência, pois promove o desenvolvimento de competências e da autonomia para o trabalho pelo conhecimento ${ }^{(6)}$.

Resultados de um estudo realizado em Porto Alegre (RS), Brasil, destacam a utilização da EPS como uma possibilidade de abertura para novos modos de saber e novos modos de fazer em enfermagem. O modelo utilizado permitiu melhoria da formação dos profissionais, ressoando na segurança e na qualidade da assistência. Nesse sentido, um programa de educação permanente no contexto hospitalar poderá ajudar coletivamente, pois reforça as aprendizagens significativas e amplia a possibilidade de mudanças positivas no serviço ${ }^{(7)}$.

Diante da destacada importância da educação permanente para a saúde e para enfermagem, da problemática da formação ainda focada no modelo biomédico e em práticas tradicionais 
de educação, decidiu-se pela realização desta pesquisa que tem como objetivo analisar as repercussões das atividades de educação permanente nas práticas assistenciais dos profissionais de enfermagem.

\section{Metodologia}

Trata-se de uma pesquisa qualitativa, realizada em um hospital universitário (HU) de grande porte, referência em média e alta complexidade, localizado no município de Salvador, Bahia, Brasil. O HU é uma organização credenciada pelo Ministério da Educação (MEC) como hospital de ensino. Dentre suas missões está a prestação de assistência à saúde da população; formação de recursos humanos para práticas de ensino, pesquisa e assistência; e produção de conhecimentos em benefício da coletividade.

Os participantes do estudo foram 17 profissionais de enfermagem do $\mathrm{HU}$, sendo 11 técnicas em enfermagem e 6 enfermeiras. A escolha dos participantes foi aleatória, procurando-se abranger as diferentes unidades assistenciais, categorias, turnos de trabalho e disponibilidade para participar da pesquisa. O tamanho da amostra foi definido de acordo com os critérios de saturação teórica das respostas, quando se identificou a possibilidade de generalização das respostas para o grupo pesquisado ${ }^{(8)}$. Esses profissionais foram identificados por meio do acesso ao banco de dados do projeto intitulado "Repercussões de Capacitações da Enfermagem nas Práticas Assistenciais de um Hospital Universitário”, financiado pelo Programa Permanecer da Universidade Federal da Bahia, Edital n. 2/2017, cujos profissionais atenderam aos seguintes critérios de inclusão: ter participado de pelo menos duas capacitações promovidas pela Comissão de Educação Permanente em Enfermagem (CEPE) do HU, entre agosto de 2016 e março de 2017; e possuir, no mínimo, seis meses completos de exercício profissional na instituição.

A CEPE tem um calendário permanente de capacitações voltado para a equipe de enfermagem, com a realização, em média, de cinco atividades por mês, incluindo aulas, cursos, sessões científicas e seminários. No período citado, 286 profissionais da enfermagem foram classificados como elegíveis, de acordo com os critérios de inclusão citados. Foram excluídos os profissionais que se encontravam de licença médica ou maternidade, que foram aposentados ou desligados da organização e os que participavam da CEPE na qualidade de membro ou instrutor.

A coleta de dados foi realizada entre os meses de setembro e dezembro de 2017, por meio de entrevistas semiestruturadas, realizadas em local privativo, de modo a evitar interferências externas e garantir o sigilo e a privacidade dos participantes. O instrumento de coleta de dados contemplou informações sobre sexo, categoria profissional e número de atividades de educação permanente das quais participou. Contemplou também questões norteadoras sobre as repercussões da educação permanente nas práticas assistenciais e os fatores intervenientes na aplicação dos conhecimentos adquiridos.

As entrevistas foram transcritas na íntegra, conferidas, codificadas e adequadas às regras do software IRAMUTEQ (Interface de $R$ pour les Analyses Multidimensionnelles de Textes et de Questionnaires), utilizado para o processamento dos dados. Este software é licenciado por GNU GPL (v2) utilizado para análises estatísticas sobre dados textuais e tabelas com informações sobre pessoas ou palavras. Trata-se de um programa gratuito e de fonte aberta, desenvolvido por Pierre Ratinaud em 2009. A análise com a utilização desse software tem várias finalidades, sendo possível analisar textos, entrevistas, documentos, redações, dentre outros materiais textuais $^{(9)}$, mostrando-se relevante à análise de matéria que expressa conteúdo simbólico, como pensamentos, crenças e opiniões ${ }^{(10)}$.

O corpus da pesquisa, formado pelas 17 entrevistas, foi organizado em arquivo único e conforme as normas do IRAMUTEQ. Após o processamento pelo software, obteve-se, pelo método da Classificação Hierárquica Descendente (CHD), 5 classes e 320 Unidades de Contexto Elementar (UCE). Para a identificação das entrevistas, empregou-se a letra $\mathrm{E}$ seguida do número de ordem de realização e da categoria profissional 
do participante (Enf. para enfermeiras e TE para técnicas em enfermagem).

A pesquisa respeitou os princípios éticos contidos na Resolução n. 466/2012 do Conselho Nacional de Saúde, tendo sido apreciada por um Comitê de Ética em Pesquisa mediante emissão de CAAE n. 66018217.8.0000.0049. Os profissionais de enfermagem que participaram do estudo assinaram o Termo de Consentimento Livre e Esclarecido, após serem esclarecidos quanto ao objetivo da pesquisa, bem como acerca dos aspectos éticos e legais que envolvem o estudo com seres humanos.

\section{Resultados}

Dos entrevistados, a maioria pertence ao sexo feminino (16), 11 são técnicas em enfermagem e 6 enfermeiras. Houve predominância de profissionais com mais de um ano de trabalho no campo da pesquisa e que participaram de mais de duas capacitações no período considerado no estudo.

Do corpus da pesquisa processado pelo IRAMUTEQ, obteve-se um percentual de retenção do texto de 80,3\%. As UCE foram organizadas automaticamente, de acordo com o método da Classificação Hierárquica Descendente (CHD), em cinco classes diferentes, conforme a Figura 1.

Figura 1 - Dendograma da Classificação Hierárquica Descendente (CHD) do corpus da pesquisa, Repercussões da Educação Permanente nas Práticas Assistenciais da Enfermagem. Salvador, Bahia, Brasil - 2018

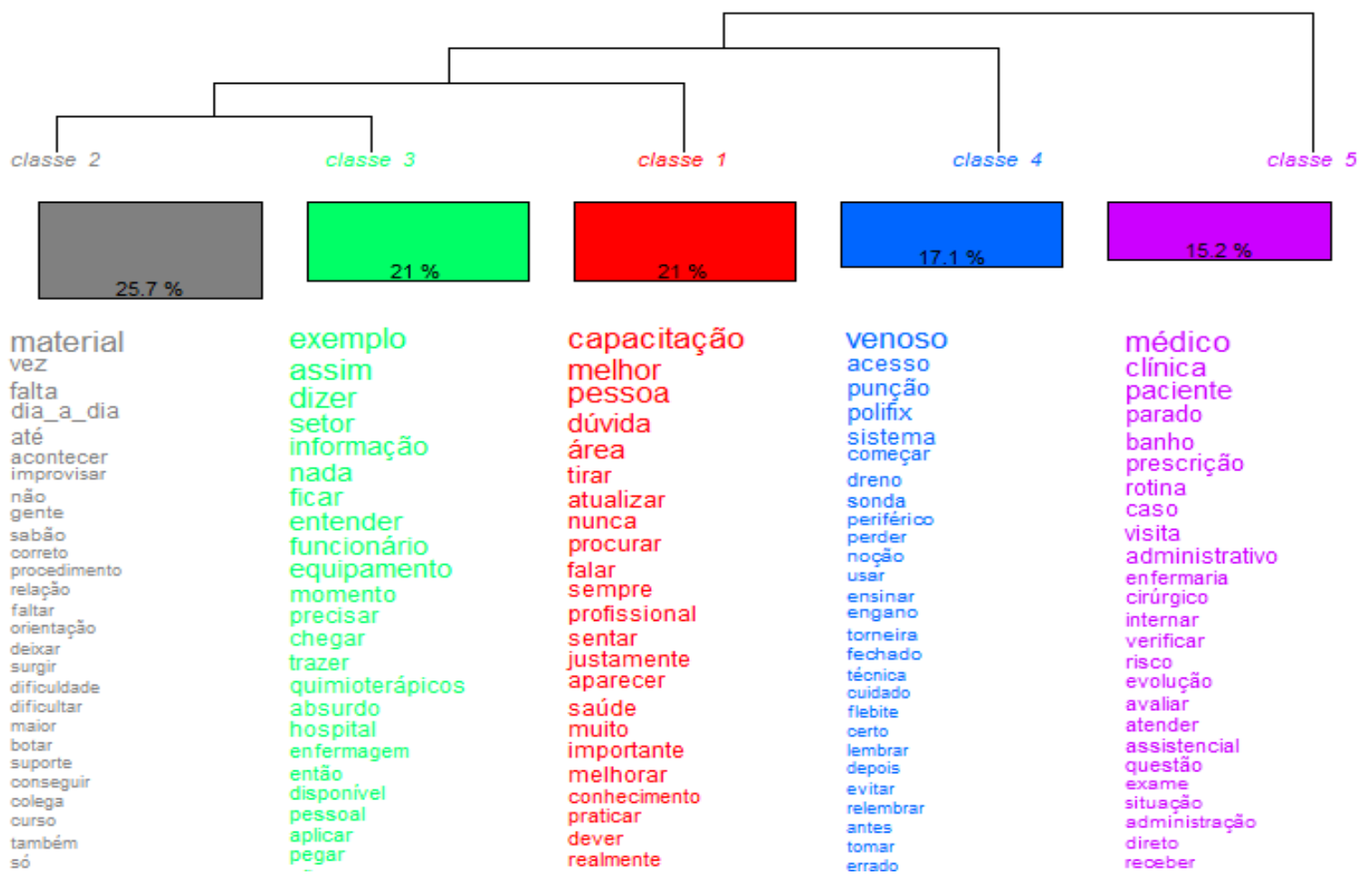

Fonte: Elaboração própria.

A classe 1 contém 21\% das UCE retidas pelo IRAMUTEQ. Traz em destaque a palavra capacitação, que representa, em associação com as demais palavras classificadas, a ideia de que as capacitações tiram dúvidas e atualizam o profissional, sendo importantes por permitir o acesso ao conhecimento e melhorar a prática. Assim, constitui-se fator muito importante para a segurança do paciente. A leitura e interpretação de seu conteúdo tornou possível identificar-se que está voltada para as repercussões da participação da equipe de enfermagem nas atividades 
de educação permanente nas práticas assistenciais. Esta classe expressa um conteúdo diretamente relacionado ao objeto em estudo.

De acordo com as falas das participantes, as atividades promovidas são um instrumento importante para o exercício do cuidado. Dentre os qualificadores para as atividades, como pode ser verificado na Figura 1, destacaram-se expressões, como: atualiza, tira dúvidas, renova, capacita e relembra. Os excertos a seguir são ilustrativos.

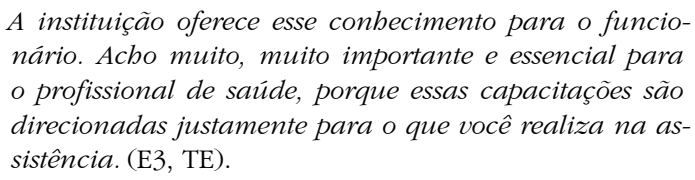

Elas foram muito importantes e, quando aparecer, eu vou continuar fazendo. Só faz enriquecer o nosso conhecimento e a gente praticar com segurança, prestar um melhor atendimento ao cliente. Sim, a minha prática melhorou principalmente em ECG [eletrocardiograma]. (E5, TE).

Ainda de acordo com as participantes, as capacitações são lembradas como fundamentais para a realidade do trabalho. As repercussões citadas vão na direção de ajudar os profissionais a prestar uma assistência mais qualificada. As metodologias utilizadas nas aulas parecem constituir-se elementos facilitadores, uma vez que são abertas, sem uma hierarquia rígida de saberes e há liberdade para questionamentos e esclarecimentos de dúvidas.

O bom disso tudo é que a pessoa se sente livre para questionar, para buscar, para entender como está aquele lugar. Eu estou com essa dúvida aqui, não vou aplicar dessa forma, vou procurar, vou falar com alguém. (E11, TE).

Ai fica mais fácil, porque você não se sente incomodado em ter uma dúvida; você não se sente inferiorizado porque não domina. Eu acho isso muito importante. (E11, TE).

Eu, sinceramente, gosto muito, porque conbecimento nunca é demais, e o pessoal que faz o curso, eles realmente se preocupam muito em passar o máximo possível de uma maneira clara e específica que acaba acrescentando muito para a gente. (E13, TE).

A classe 2 contém 25,7\% das UCE retidas pelo IRAMUTEQ e traz o material médico-hospitalar como palavra em destaque. Os participantes enfatizaram a importância dos materiais no dia a dia para a realização dos procedimentos assistenciais. Também foi lembrada a dificuldade para promover a assistência devido à escassez de recursos materiais, podendo divergir da orientação fornecida e comprometer, algumas vezes, a qualidade da assistência. Assim, os profissionais veem-se obrigados a lançar mão do improviso, uma das palavras em destaque nesta classe. Falta material. A gente não tem condições de manter
uma rotina de assistência perfeita como deveria ser,
como uma técnica extremamente correta. Então faltam
pequenas coisas que garantiriam uma melhor assistên-
cia. (E10, Enf).

A técnica que às vezes a gente não faz corretamente devido ao dia a dia, à correria, e às vezes também falta muito material e ajuda a gente não fazer as coisas corretas. (E2, TE).

[...] por exemplo, tem o déficit de material e de outras coisas que comprometem. (E9, TE).

A classe 3 contém 21\% das UCE retidas pelo IRAMUTEQ e traz a palavra exemplo como destaque. Essa classe expõe os fatores intervenientes para a implementação dos conhecimentos adquiridos com a participação nas capacitações. Para os participantes, vários fatores interferem na implementação dos conhecimentos, como o remanejamento dos profissionais para unidades assistenciais diferentes das que estão lotados, com a necessidade de adaptação à rotina e à dinâmica em outros setores; dificuldades administrativas, como a existência de equipamentos diversos para a mesma função e o manuseio correto de novos equipamentos; divergência de consenso entre a equipe quanto às rotinas assistenciais; o fato de as enfermeiras assumirem muitas funções administrativas e se distanciarem da assistência direta aos pacientes; dificuldades na relação com a equipe médica; e o fato de as capacitações oferecidas não serem específicas para profissionais de nível técnico.

\footnotetext{
Então acaba, como se diz, assim, é descobrindo um santo para cobrir o outro; inclusive, semana passada, aconteceu isso comigo. Tiraram funcionário daqui e botaram em outra unidade. A daqui ficou sobrecarregada. (E1, TE).

Eu, por exemplo, adoro ouvir meus pacientes. Fico revoltada no dia que eu estou sobrecarregada e não tenbo como dar o carinho e a atenção que eles merecem. E acho que aqui nós damos mesmo. (E13, TE).
}

A classe 4 contém um percentual de 17,1\% das UCE retidas pelo IRAMUTEQ. Em seu conteúdo, traz os temas das capacitações que foram mais lembrados pelos participantes por possuírem 
estreita relação com a assistência - acesso venoso, com seus cuidados, possibilidades de complicações e dispositivos necessários para manter e utilizar o sistema; sondas e drenos; e eletrocardiograma (ECG). Outro aspecto destacado é a preocupação com a segurança, para evitar erros e enganos, além da possibilidade de danos aos pacientes. Os participantes também ressaltaram temas de capacitações que poderiam ser repetidos e a necessidade de revisão de técnicas assistenciais.

A gente vai perdendo alguns cuidados e tudo. E esses en-
contros acabam fazendo a gente relembrar e rever algu-
mas práticas e algumas atitudes. Então, são importantes,
sempre! Em algumas vezes, eu consigo conciliar o traba-
lho e essas capacitações. (E6, Enf).

E através da capacitação a gente aprende isso no curso, a gente aprende realmente a ter uma noção do que é o certo e do que é errado na enfermagem. (E8, TE).

Tem melhorado, devido a alguns detalhes que acabam nos alertando sobre as boas práticas. A [aula] de punção venosa mesmo, a gravatinha que orientou a gente fazer foi muito boa e todos os cursos só têm a acrescentar. (E13, TE).

Então era para ter sempre sobre medicação, sobre dreno, sobre sondas e sobre várias coisas e protocolos da unidade [...] (E4, TE).

[...] a questão do que a gente tem muito aqui que éflebite. Então a gente tomou aquele curso de acesso venoso, que foi muito bom; o curso foi um dos melhores. (E10, TE).

As meninas também tiveram mais atenção na higienização, na desinfecção no momento de fazer assepsia para puncionar, no momento do manuseio. Então, assim, foi bastante produtiva essa capacitação de acesso venoso periférico. (E16, Enf).

A classe 5 representa um percentual de 15,2\% das UCE. Traz como palavra de destaque o médico e o quadro clínico do paciente. Ao se interpretar o conteúdo, identificam-se os diferentes pontos de vista dos profissionais de enfermagem a respeito das práticas dos médicos em comparação à atribuição das enfermeiras. Os discursos dos participantes que compuseram esta classe, em sua maioria enfermeiras, revelaram uma prática especialmente voltada para a rotina diária de assistência ao paciente. Em suas falas, as enfermeiras deixaram transparecer a necessidade de, além de assistir ao paciente, supervisionar os técnicos e gerenciar a dinâmica da unidade. Assim, revelaram a dubiedade existente entre a demanda do trabalho e a responsabilidade, com repercussões tanto no gerenciamento quanto na assistência.

Nós, a parte da enfermagem, além de ter que dar uma
assistência ao paciente, ainda temos que ficar fazendo
a parte administrativa, levar paciente para fazer exame,
ir atrás de médico para carimbar a prescrição. (E1, TE).

Fazer as visitas; olhar os pacientes resumidamente; fazemos os curativos e observamos também a questão do pessoal, dos técnicos de enfermagem, pois nós somos responsáveis por eles e nós fazemos a distribuição, atendemos às solicitações dos técnicos de enfermagem, que eles vão nos colocando na enfermaria. (E12, Enf).

Mas, assim, o médico é visto como o principal, o protagonista, porque é ele que vai avaliar o paciente para prescrever [...] medicação, tratamento e, principalmente, com eles que a comunicação às vezes falha e não tem porque ser assim. (E15, Enf).

\section{Discussão}

De acordo com os relatos dos entrevistados, as repercussões das capacitações indicam diversos pontos positivos, como melhoria na qualidade da assistência, esclarecimento de dúvidas do dia a dia e atualização quanto às práticas corretas. Resultados semelhantes foram divulgados por pesquisa internacional realizada na Espanha, que identificou o aprimoramento na assistência de enfermagem após participação dos profissionais em atividades de educação ${ }^{(11)}$. Estes dados também vão ao encontro de um estudo realizado em Minas Gerais, Brasil, no qual os participantes indicaram que as atividades de educação permanente possibilitaram a aquisição de conhecimentos úteis para a atuação profissional. Nessa pesquisa, os participantes reconheceram que o espaço propiciado pelas ações de educação permanente favoreceu a atualização, o compartilhamento de experiências, a construção de novas relações e constituiu-se como potencializador de um jeito novo de trabalhar ${ }^{(12)}$.

Os resultados desta pesquisa apresentam confluência com outro estudo que aponta a educação permanente como uma ferramenta útil para melhorar a realidade, ao permitir a reflexão sobre o cotidiano, com suas dificuldades e potencialidades ${ }^{(13)}$. Assim, ao ser planejada com base em questionamentos levantados na realidade, fortalece-se como uma importante 
ferramenta para o trabalho e contribui para a melhoria da qualidade dos serviços de saúde ${ }^{(12)}$.

A educação permanente é discutida como um meio que favorece uma aprendizagem significativa e transformadora na área da saúde. Um modelo de gestão democrático e descentralizado também favorece tal transformação ${ }^{(14)}$. Com isso, os profissionais conseguem interagir com a gestão, o que beneficia a todos no ambiente de trabalho. Traz ainda pontos positivos para a qualificação e a segurança do profissional, favorecendo a qualidade da assistência ao paciente.

A educação permanente, por ser uma das estratégias mais efetivas da qualificação de profissionais da saúde, perpassa diversos fatores, como a estrutura e a organização dos serviços, atualização sobre novas tecnologias, além de disponibilidade de materiais, equipamentos e medicações pela instituição ${ }^{(15)}$. Essas informações também estão em consonância com os resultados desta pesquisa, uma vez que os participantes destacaram as dificuldades gerenciais, administrativas, estruturais e, principalmente, a falta de materiais como fatores negativos intervenientes à implementação dos conhecimentos adquiridos nas capacitações.

Pesquisa qualitativa realizada em um hospital universitário do Rio Grande do Sul, Brasil, sobre a gestão de qualidade, traz em seus resultados as dificuldades na gestão da qualidade da assistência, cujos achados se entrelaçam com os relatos obtidos nesta pesquisa. Ficou demonstrado que a disponibilidade de materiais e equipamentos de suporte qualifica a assistência, já que a falta implica negativamente na qualidade do serviço oferecido, dificultando a execução de ações de forma segura e efetiva. Além da quantidade, a qualidade dos materiais também é necessária para atender às necessidades e evitar possíveis danos à saúde dos pacientes, a exemplo dos riscos de infecção e a repetição de procedimentos dolorosos, como a punção venosa ${ }^{(16)}$. Desta forma, reforça-se a importância de evitar as adequações, adaptações e substituições do material ideal por meio do improviso.

Os temas das capacitações lembrados pelos entrevistados dão conta do quão o conhecimento adquirido está imbrincado com a prática e de como é útil para o cotidiano profissional. Todos os assuntos estão relacionados com a realidade do trabalho e com as atribuições do profissional de enfermagem, como relatam nas entrevistas. Assim, acredita-se que a confluência entre a teoria e a prática pode servir como motivação para o trabalho e repercutir positivamente na qualidade da assistência. Nessa perspectiva, pesquisa desenvolvida no país retrata a importância da educação permanente como agente qualificador da prática profissional ${ }^{(17)}$.

A educação permanente em saúde tem grande importância na qualificação dos profissionais, devido à abordagem de temas com o objetivo de sanar as dúvidas e os problemas encontrados na realidade do trabalho. Consequentemente, pode trazer benefícios para a qualidade da assistência e qualificação dos trabalhadores ${ }^{(12)}$.

A interdependência entre a abordagem do trabalho e a educação na saúde possibilita influenciar nos temas das capacitações, retoma e observa o trabalho como princípio educativo abordado com base na perspectiva da educação interprofissional. A prática e os fatores que influenciam na qualidade da assistência necessitam ser debatidos pela equipe multiprofissional ${ }^{(18)}$.

A divisão técnica faz parte do processo de trabalho na enfermagem. Nessa direção, o discurso das participantes reproduziu tal divisão. Houve diferenças entre as falas das enfermeiras e as das técnicas em enfermagem. Enquanto as técnicas manifestaram satisfação com as capacitações mais voltadas para os aspectos do dia a dia e com o fato de os assuntos abordados estarem relacionados com a prática, as enfermeiras, por sua vez, apresentaram um discurso permeado pela rotina de trabalho nas unidades assistenciais. Assim, deixaram transparecer as diferenças nas características laborais e seu papel enquanto coordenadoras do processo de trabalho em suas interfaces com outras profissões, como a medicina $^{(19)}$.

Ainda ficaram evidentes, de acordo com o conteúdo disposto na classe 5 , as dificuldades enfrentadas pelos profissionais de enfermagem, no que diz respeito à organização do processo 
de trabalho em benefício do cuidado ao paciente. Segundo os participantes, é necessário ir em busca do médico para fazer o que é de sua atribuição, como carimbar uma prescrição. O médico também é retratado em uma posição de protagonismo na assistência ao paciente, e assim fica evidente a falta de reconhecimento da enfermeira e da importância do seu trabalho. Defende-se que, em uma equipe de assistência à saúde, o protagonismo é de todos os membros. Sem os esforços individuais e coordenados, o objetivo final de prestar o melhor cuidado e restabelecer a saúde do paciente não é alcançado.

Apresenta-se como limite da pesquisa o fato ter sido realizada com diferentes categorias dentro da enfermagem, sem permitir estabelecer uma delimitação clara entre as repercussões da participação em atividades de educação permanente para as técnicas em enfermagem e as enfermeiras. Além disso, acredita-se que seria importante pesquisar também entre profissionais que participaram de maior número de capacitações e com um distanciamento temporal maior, para se evidenciar a permanência das mudanças afirmadas pelos participantes, indicar necessidades de mudanças nas estratégias do planejamento da educação permanente, ou, até mesmo, realizar uma comparação de aprendizagem entre quem participou de mais capacitações e quem participou menos, sendo outra forma de observar as mudanças na prática. No entanto, os resultados encontrados são suficientes para afirmar que a educação permanente tem se constituído como uma importante estratégia de suporte à qualificação para o trabalho e como elemento de apoio à qualidade dos cuidados de enfermagem.

\section{Conclusão}

A análise das repercussões da participação em atividades de educação permanente nas práticas assistenciais da enfermagem permitiu identificar que a possibilidade de esclarecimento de dúvidas, de atualização, a aquisição de conhecimentos e as melhorias sentidas na segurança para o exercício profissional e na qualidade da assistência prestada constituíram-se em elementos positivos para os entrevistados.

Fatores como a falta de materiais ou a aquisição de materiais de baixa qualidade foram destacados pela possibilidade de interferência negativa na assistência, por não permitirem a execução correta de técnicas, exigir criatividade para adaptações e improvisos e, assim, dificultar a implementação dos conhecimentos adquiridos.

Nota-se que há adesão e participação dos profissionais no processo de educação permanente, pois se mostraram sensíveis, ávidos por conhecimentos e motivados a participar, mesmo diante de dificuldades gerenciais, administrativas, estruturais e das exigências das rotinas, que geram certo distanciamento da assistência direta aos pacientes.

As capacitações realizadas estavam diretamente relacionadas com a realidade vivida pela equipe de enfermagem, sendo lembradas como importantes para a prática. Conforme descrito, reverberam na qualidade da assistência. Assim, pode-se concluir que, para os profissionais de enfermagem, a participação em atividades de educação permanente tem repercussões positivas na qualidade da assistência.

\section{Colaborações:}

1. concepção, projeto, análise e interpretação dos dados: Valdenir Almeida da Silva, Rosana Santos Mota e Andreia Santos Mendes;

2. redação do artigo e revisão crítica relevante do conteúdo intelectual: Maristela Conceição de Jesus, Valdenir Almeida da Silva, Rosana Santos Mota, Juliana Cana Brazil Costa, Andreia Santos Mendes e Marcelle de Jesus Oliveira;

3. aprovação final da versão a ser publicada: Valdenir Almeida da Silva e Rosana Santos Mota.

\section{Referências}

1. World Health Organization. Transforming and scaling up health professionals' education and training. Geneva; 2013 [Internet]. [cited 2018 Jan 18]. Available from: http://apps.who.int/iris/ bitstream/10665/93635/1/9789241506502_eng. pdf?ua $=1$ 
2. Brasil. Ministério da Saúde. Secretaria de Gestão no Trabalho e da Educação na Saúde. Departamento de Gestão da Educação em Saúde. Política Nacional de Educação Permanente em Saúde. Brasília, DF; 2009. [Internet]. [cited 2018 Jan 18]. Available from: http://portal. anvisa.gov.br/documents/33856/396770/ Pol\%C3\%ADtica+Nacional+de+Educa $\%$ C3\%A7\% $\mathrm{C} 3 \% \mathrm{~A} 3 \mathrm{O}+$ Permanente $+\mathrm{em}+\mathrm{Sa} \% \mathrm{C} 3 \% \mathrm{BAde} /$ c92db117-e170-45e7-9984-8a7cdb111faa

3. Silva CT, Terra MG, Camponogara S, Kruse MHL, Roso CC, Xavier MS. Educação permanente em saúde a partir de uma residência multidisciplinar: estudo de caso. Rev gaúcha enferm [Internet]. 2014 set [cited 2018 Jan 18];35(3):49-54. Available from: www.scielo.br/scielo.php?pid=S1983-1447201 4000300049\&script=sci...tlng...

4. Díaz VB, Martínez MR, Figueredo ZCA, Solis FR. La superación continua de enfermeira y su contribución al desempeño de excelencia. Rev Cubana Enferm [Internet]. 2017 ene-mar [cited 2018 Jan 18];33(1). Available from: http://www. revenfermeria.sld.cu/index.php/enf/article/ view/1095/234

5. Miccas FL, Batista SHSS. Educação permanente em saúde: metassíntese. Rev saúde pública [Internet]. 2014 [cited 2018 Jan 18];48(1):170-85. Available from: http://dx.doi.org/10.1590/S00348910.2014048004498

6. Lima LPS, Ribeiro MRR. A competência para Educação Permanente em Saúde: percepções de coordenadores de graduações da saúde. Physis Rev Saúde Coletiva [Internet]. 2016 [cited 2018 Jan 18];26(2):483-501. Available from: http://dx.doi. org/10.1590/S0103-73312016000200008

7. Flores GE, Oliveira DLL, Zocche DAA. Educação permanente no contexto hospitalar: a experiência que ressignifica o cuidado em enfermagem. Trab educ saúde [Internet]. 2016 abr [cited 2018 Jan 18];14(2):487-504. Available from: www.scielo.br/scielo.php?pid=\$1981-7746201 6000200487\&script=sci_abstract

8. Nascimento LCN, Souza TV, Oliveira ICS, Moraes JRMM, Aguiar RCB, Silva LF. Theoretical saturation in qualitative research: an experience report in interview with schoolchildren. Rev Bras Enferm [Internet]. 2018 [cited 2018 Jan 18];71(1):228-33. Available from: DOI: http://dx.doi.org/10.1590/ 0034-7167-2016-0616

9. Camargo BV, Justo AM. Tutorial para uso do software de análise textual IRAMUTEQ
[Internet]. Universidade Federal de Santa Catarina. Laboratório de Psicologia Social da Comunicação e Cognição. Florianópolis: LACCOS; 2013. [cited 2018 Jan 18]. Available from: www.iramuteq.org/ documentation/fichiers/tutoriel-en-portugais

10. Camargo BV, Justo AM. IRAMUTEQ: um software gratuito para análise de dados textuais. Temas Psicol [Internet]. 2013 [cited 2018 Jan 18];21(2):513-8. Available from: http://dx.doi. org/10.9788/TP2013.2-16

11. Peña García I, López Vallecillo M, Barrios Díez E, Sánchez Sánchez S, Moreno Cea L, Jiménez Mayoral A. Evaluación del impacto de la formación continuada de enfermería a través de un video tutorial sobre el registro de valoración del paciente. Tesela [Rev Tesela] [Internet]. 2017 [cited 2018 Jan 18];21. Available from: http://www. index-f.com/tesela/ts21/ts10441.php

12. Silva KL, Matos JAV, França BD. A construção da educação permanente no processo de trabalho em saúde no estado de Minas Gerais, Brasil. Esc Anna Nery [Internet]. 2017 [cited 2018 Jan 2018];21(4):e20170080. Available from: DOI: 10.1590/2177-9465-EAN-2017-0060

13. Moraes KG, Dytz JLG. Política de Educação Permanente em Saúde: análise de sua implementação. ABCS Health Sci [Internet]. 2015 [cited 2018 Jan 18];40(3):263-9. Available from: https://www.portalnepas.org.br/abcshs/article/ view/806

14. Campos KFC, Sena RR, Silva KL. Educação permanente nos serviços de saúde. Esc Anna Nery [Internet]. 2017 [cited 2018 Jan 20];21(4):e20160317. Available from: DOI: 10.1590/2177-9465-EAN-2016-0317

15. Silva LAA, Schmidt SMS, Noal HC, Signor E, Gomes IEM. Avaliação da educação permanente no processo de trabalho em saúde. Trab Educ Saúde [Internet]. 2016 set/dez [cited 2018 Jan 16];14(3):765-81. Available from: http://dx.doi. org/10.1590/1981-7746-sol00015

16. Rabenschlag LA, Lima SBS, Eberhardt TD, Kessler M, Soares RSA, Camponogara S. Gestão da qualidade na assistência de enfermagem em unidades de clínica cirúrgica. Rev Enferm UFSM [Internet]. 2015 abr/jun [cited 2018 Jan 18];5(2):235-46. Available from: https://periodicos.ufsm.br/reufsm/article/ view/15891

17. Mota RS, Silva VA, Costa JCB, Barros A, Gomes BP, Araújo RPA. Participation in continuing education 
activities and changes in nursing care practices. Rev baiana enferm [Internet]. 2018 [cited 2018 Jan 18];32:e26485. Available from: DOI 10.18471/rbe. v32.26485

18. Peduzzi M. Trabalho e educação na saúde: ampliação da abordagem de recursos humanos. Ciênc Saúde Colet [Internet]. 2013 [cited 2018 Jan 18];18(6):1535-43. Available from: www.scielo.br/scielo.php?script=sci_arttext\& pid=\$1413-81232013000600005
19. Leal JAL, Melo CMM. Processo de trabalho da enfermeira em diferentes países: uma revisão integrativa. Rev Bras Enferm [Internet]. 2018 [cited 2018 Jan 18];71(2):441-52. Available from: http:// dx.doi.org/10.1590/0034-7167-2016-0468

Recebido: 5 de agosto de 2018 Aprovado: 12 de fevereiro de 2019

Publicado: 18 de junho de 2019

A Revista Baiana de Enfermagem utiliza a Licença Creative Commons - Atribuição-NãoComercial 4.0 Internacional. https://creativecommons.org/licenses/by-nc/4.0/ Este artigo é de acesso aberto distribuído sob os termos da Licença Creative Commons (CC BY-NC). Esta licença permite que outros remixem, adaptem e criem a partir do seu trabalho para fins não comerciais. Embora os novos trabalhos tenham de lhe atribuir o devido crédito e não possam ser usados para fins comerciais, os usuários não têm de licenciar esses trabalhos derivados sob os mesmos termos. 